Bull. Korean Math. Soc. 46 (2009), No. 6, pp. 1091-1097

DOI 10.4134/BKMS.2009.46.6.1091

\title{
EXTENSIONS OF GENERALIZED STABLE RINGS
}

\author{
ZHANG WANRU
}

\begin{abstract}
In this paper, we investigate the extensions of generalized stable rings. It is shown that a ring $R$ is a generalized stable ring if and only if $R$ has a complete orthogonal set $\left\{e_{1}, \ldots, e_{n}\right\}$ of idempotents such that $e_{1} R e_{1}, \ldots, e_{n} R e_{n}$ are generalized stable rings. Also, we prove that a ring $R$ is a generalized stable ring if and only if $R[[X]]$ is a generalized stable ring if and only if $T(R, M)$ is a generalized stable ring.
\end{abstract}

\section{Introduction}

Let $R$ be an associative ring with identity. A ring $R$ is said to have stable range one provided that $a R+b R=R$ with $a, b \in R$ implies that there exists $y \in R$ such that $a+b y \in U(R)$, where $U(R)$ is the set of all units in $R$. This definition is left-right symmetric. Moreover, we know that a right $R$-module $M$ can be cancelled from direct sums if and only if $\operatorname{End}_{R}(M)$ has stable range one. Many authors have studied stable range one conditions and its generalizations from different viewpoints such as $[1,3-12,14]$.

As a generalization of rings with stable range one, in [9], Chen introduced the generalized stable rings. A ring $R$ is called a generalized stable ring provided that $a R+b R=R$ with $a, b \in R$ implies that there exists $y \in R$ such that $a+b y \in K(R)$, where $K(R)=\{x \in R \mid$ there exist $s, t \in R$ such that $s x t=$ $1\}$. Obviously, if a ring $R$ has stable range one, then it is a generalized stable ring, but the converse is not true, see [9, Example 3]. Thus generalized stable rings are nontrivial generalizations of rings with stable range one. For more details and examples of generalized stable rings, see $[5,6,9]$.

In this paper, we investigate the extensions of generalized stable rings. It is shown that a ring $R$ is a generalized stable ring if and only if $R$ has a complete orthogonal set $\left\{e_{1}, \ldots, e_{n}\right\}$ of idempotents such that $e_{1} R e_{1}, \ldots, e_{n} R e_{n}$ are generalized stable rings. Also, we prove that a ring $R$ is a generalized stable ring if and only if $R[[X]]$ is a generalized stable ring if and only if $T(R, M)$ is a generalized stable ring.

Received September 3, 2008.

2000 Mathematics Subject Classification. 16E50, 16 U99.

Key words and phrases. generalized stable rings, complete orthogonal set of idempotents, ring extensions. 


\section{Main results}

The following result appeared in [9, Theorem 9].

Lemma 2.1. Let $A$ be a right $R$-module, $E=\operatorname{End}_{R}(A)$ be its endomorphism ring. Then the following are equivalent:

(1) E is a generalized stable ring.

(2) Given any decompositions $M=A_{1} \oplus B_{1}=A_{2} \oplus B_{2}$ with $A_{1} \cong A \cong A_{2}$, there exist $C, D, E \leq M$ such that $M=C \oplus D \oplus B_{1}=C \oplus E \oplus B_{2}$ with $C \cong A$.

Lemma 2.2. Let $M_{1}, M_{2}, \ldots, M_{n}$ be right $R$-modules. If $\operatorname{End}_{R}\left(M_{1}\right), \operatorname{End}_{R}\left(M_{2}\right)$, $\ldots, \operatorname{End}_{R}\left(M_{n}\right)$ are generalized stable rings, then so is $\operatorname{End}_{R}\left(M_{1} \oplus M_{2} \oplus \cdots \oplus\right.$ $\left.M_{n}\right)$.

Proof. Given right $R$-module decompositions $M=A_{1} \oplus B=A_{2} \oplus C$ with $A_{1} \cong M_{1} \oplus M_{2} \oplus \cdots \oplus M_{n} \cong A_{2}$, then we have $A_{1}=A_{11} \oplus A_{12} \oplus \cdots \oplus A_{1 n}$ and $A_{2}=A_{21} \oplus A_{22} \oplus \cdots \oplus A_{2 n}$ with $A_{1 i} \cong M_{i} \cong A_{2 i}$ for $1 \leq i \leq n$. So $M=A_{11} \oplus A_{12} \oplus \cdots \oplus A_{1 n} \oplus B=A_{21} \oplus A_{22} \oplus \cdots \oplus A_{2 n} \oplus C . \operatorname{Since}_{\operatorname{End}}\left(M_{1}\right)$ is a generalized stable ring and $A_{11} \cong M_{1} \cong A_{21}$, by Lemma 2.1, we can find submodules $C_{1}, D_{1}, E_{1} \leq M$ such that $M=C_{1} \oplus D_{1} \oplus A_{12} \oplus \cdots \oplus A_{1 n} \oplus B=$ $C_{1} \oplus E_{1} \oplus A_{22} \oplus \cdots \oplus A_{2 n} \oplus C$ with $C_{1} \cong M_{1}$. Likewise, we have submodules $C_{2}, \ldots, C_{n}, D_{2}, \ldots, D_{n}, E_{2}, \ldots, E_{n}$ such that $M=C_{1} \oplus \cdots \oplus C_{n} \oplus D_{1} \oplus \cdots \oplus$ $D_{n} \oplus B=C_{1} \oplus \cdots \oplus C_{n} \oplus E_{1} \oplus \cdots \oplus E_{n} \oplus C$ with $C_{i} \cong M_{i}$ for $1 \leq i \leq n$. Clearly, $C_{1} \oplus \cdots \oplus C_{n} \cong M_{1} \oplus \cdots \oplus M_{n}$. By Lemma 2.1, we conclude that $\operatorname{End}_{R}\left(M_{1} \oplus M_{2} \oplus \cdots \oplus M_{n}\right)$ is a generalized stable ring.

A finite orthogonal set of idempotents $e_{1}, \ldots, e_{n}$ in a ring $R$ is said to be complete in case $e_{1}+\cdots+e_{n}=1 \in R$.

Theorem 2.3. The following conditions are equivalent:

(1) $R$ is a generalized stable ring.

(2) There exists a complete orthogonal set $\left\{e_{1}, \ldots, e_{n}\right\}$ of idempotents such that $e_{1} R e_{1}, \ldots, e_{n} R e_{n}$ are generalized stable rings.

Proof. (1) $\Rightarrow(2)$ is obvious.

$(2) \Rightarrow(1)$ It follows by $e_{i} R e_{i} \cong \operatorname{End}_{R}\left(e_{i} R\right)$ and Lemma 2.2 that $\operatorname{End}_{R}\left(e_{1} R \oplus\right.$ $\left.\cdots \oplus e_{n} R\right)$ is a generalized stable ring. One easily checks that $R=e_{1} R \oplus \cdots \oplus$ $e_{n} R$. Hence $R \cong \operatorname{End}_{R}(R)=\operatorname{End}_{R}\left(e_{1} R \oplus \cdots \oplus e_{n} R\right)$ is a generalized stable ring.

Let $e_{1}, \ldots, e_{n}$ be idempotents of a ring $R$. Clearly, with the usual matrix operation, $\left(\begin{array}{ccc}e_{1} R e_{1} & \cdots & e_{1} R e_{n} \\ \vdots & \ddots & \vdots \\ e_{n} R e_{1} & \cdots & e_{n} R e_{n}\end{array}\right)=\left\{\left(\begin{array}{ccc}e_{1} r_{11} e_{1} & \cdots & e_{1} r_{1 n} e_{n} \\ \vdots & \ddots & \vdots \\ e_{n} r_{n 1} e_{1} & \cdots & e_{n} r_{n n} e_{n}\end{array}\right) \mid r_{i j} \in R(1 \leq i, j \leq n)\right\}$ forms a ring with the identity $\operatorname{diag}\left(e_{1}, \ldots, e_{n}\right)$. 
Proposition 2.4. Let $e_{1}, \ldots, e_{n}$ be idempotents of a ring $R$. If $e_{1} R e_{1}, \ldots$, $e_{n} R e_{n}$ are all generalized stable rings, then so is the ring

Proof. Set

$$
\left(\begin{array}{ccc}
e_{1} R e_{1} & \cdots & e_{1} R e_{n} \\
\vdots & \ddots & \vdots \\
e_{n} R e_{1} & \cdots & e_{n} R e_{n}
\end{array}\right) .
$$

$$
T=\left(\begin{array}{ccc}
e_{1} R e_{1} & \cdots & e_{1} R e_{n} \\
\vdots & \ddots & \vdots \\
e_{n} R e_{1} & \cdots & e_{n} R e_{n}
\end{array}\right) .
$$

Choose $f_{1}=\operatorname{diag}\left(e_{1}, 0, \ldots, 0\right), f_{2}=\operatorname{diag}\left(0, e_{2}, \ldots, 0\right), \ldots, f_{n}=\operatorname{diag}(0,0, \ldots$, $\left.e_{n}\right) \in T$. Then we have a complete orthogonal set $\left\{f_{1}, f_{2}, \ldots, f_{n}\right\}$ of idempotents such that $f_{i} T f_{i} \cong e_{i} R e_{i}$ are generalized stable rings. By Theorem 2.3, $T$ is a generalized stable ring.

Let $M_{n}(R)$ denotes the $n$ by $n$ matrix ring over $R$, from Proposition 2.4, we have the following result in [9].

Corollary 2.5. If $R$ is a generalized stable ring, then so is $M_{n}(R)$ for all positive integer $n$.

A Morita context denoted by $(A, B, M, N, \psi, \phi)$ consists of two rings $A, B$, two bimodules ${ }_{A} N_{B},{ }_{B} M_{A}$ and a pair of bimodule homomorphisms (called pairings) $\psi: N \otimes_{B} M \rightarrow A$ and $\phi: M \otimes_{A} N \rightarrow B$ which satisfy the following associativity: $\psi(n \otimes m) n^{\prime}=n \phi\left(m \otimes n^{\prime}\right), \phi(m \otimes n) m^{\prime}=m \psi\left(n \otimes m^{\prime}\right)$ for any $m, m^{\prime} \in M, n, n^{\prime} \in N$. These conditions ensure that the set $T$ of generalized matrices $\left(\begin{array}{cc}a & n \\ m & b\end{array}\right), a \in A, b \in B, m \in M, n \in N$ forms a ring, called the ring of the Morita context. Particularly, if $N=0$, then the Morita context is the formal triangular matrix ring.

Corollary 2.6. Let $T$ be the ring of a Morita context $(A, B, M, N, \psi, \phi)$. If $A$ and $B$ are generalized stable rings, then so is $T$.

Proof. Set $e_{1}=\left(\begin{array}{cc}1_{A} & 0 \\ 0 & 0\end{array}\right), e_{2}=\left(\begin{array}{cc}0 & 0 \\ 0 & 1_{B}\end{array}\right)$. Then $\left\{e_{1}, e_{2}\right\}$ is a complete orthogonal set of idempotents such that $e_{1} T e_{1} \cong A, e_{2} T e_{2} \cong B$ are generalized stable rings. Then we obtain the result by Theorem 2.3.

Corollary 2.7. Let $T=\left(\begin{array}{cc}A & 0 \\ M\end{array}\right)$ be the formal triangular matrix ring. If $A$ and $B$ are generalized stable rings, then so is $T$.

Theorem 2.8. Let $e_{1}, \ldots, e_{n}$ be idempotents of a ring $R$. If $e_{1} R e_{1}, \ldots, e_{n} R e_{n}$ are all generalized stable rings, then so is the ring

$$
\left(\begin{array}{cccc}
e_{1} R e_{1} & 0 & \cdots & 0 \\
e_{2} R e_{1} & e_{2} R e_{2} & \cdots & 0 \\
\vdots & \vdots & \ddots & \vdots \\
e_{n} R e_{1} & e_{n} R e_{2} & \cdots & e_{n} R e_{n}
\end{array}\right)
$$


Proof. Clearly, the result holds for $n=1$. Assume now that the result holds for $n=k \geqslant 1$. Let $n=k+1$. Set

$$
B=\left(\begin{array}{cccc}
e_{2} R e_{2} & 0 & \cdots & 0 \\
e_{3} R e_{2} & e_{3} R e_{3} & \cdots & 0 \\
\vdots & \vdots & \ddots & \vdots \\
e_{k+1} R e_{2} & e_{k+1} R e_{3} & \cdots & e_{k+1} R e_{k+1}
\end{array}\right), \quad M=\left(\begin{array}{c}
e_{2} R e_{1} \\
e_{3} R e_{1} \\
\vdots \\
e_{k+1} R e_{1}
\end{array}\right)
$$

Then $T=\left(\begin{array}{cc}e_{1} R e_{1} & 0 \\ M & B\end{array}\right)$. By hypothesis, $B$ is a generalized stable ring. Let $f_{1}=\operatorname{diag}\left(e_{1}, 0\right), f_{2}=\operatorname{diag}\left(0, \operatorname{diag}\left(e_{2}, \ldots, e_{k+1}\right)\right)$. Then $\left\{f_{1}, f_{2}\right\}$ is a complete orthogonal set of idempotents such that $f_{1} T f_{1} \cong e_{1} R e_{1}, f_{2} T f_{2} \cong B$ are generalized stable rings. Then we obtain the result by Theorem 2.3.

Corollary 2.9. If $R$ is a generalized stable ring, then so is the ring of $n \times n$ lower triangular matrices over $R$.

Analogously, we deduce that if $R$ is a generalized stable ring, then so is the ring of $n \times n$ upper triangular matrices over $R$.

In [9, Proposition 5], the author proved that a $\operatorname{ring} R$ is a generalized stable ring if and only if $R / J(R)$ is a generalized stable ring. Use the same method in $[9$, Proposition 5], we can prove the following result. We omit its proofs.

Lemma 2.10. Let $R$ be a ring, $I$ an ideal of $R$ such that $I \subseteq J(R)$. The following conditions are equivalent:

(1) $R$ is a generalized stable ring.

(2) $R / I$ is a generalized stable ring.

Let $R[[X]]$ denotes the power series ring over $R$. From Lemma 2.10, we have the following result.

Theorem 2.11. A ring $R$ is a generalized stable ring if and only if $R[[X]]$ is a generalized stable ring.

Proof. We construct a map $\phi: R[[X]] \rightarrow R$ such that $\phi\left(\sum_{i=0}^{\infty} a_{i} x^{i}\right) \rightarrow a_{0}$ for any $\sum_{i=0}^{\infty} a_{i} x^{i} \in R[[X]]$. It is easy to verify that $\operatorname{Ker} \phi=\left\{\sum_{i=0}^{\infty} a_{i} x^{i} \mid a_{0}=\right.$ $0\}$. Since for any $\Sigma_{i=0}^{\infty} a_{i} x^{i} \in \operatorname{Ker} \phi$ and $y \in R[[X]], 1-y\left(\sum_{i=0}^{\infty} a_{i} x^{i}\right)=1+$ $c_{1} x+c_{2} x^{2}+\cdots$, so $1-y\left(\sum_{i=0}^{\infty} a_{i} x^{i}\right) \in U(R[[X]])$. Hence, we have $\Sigma_{i=0}^{\infty} a_{i} x^{i} \in$ $J(R[[X]])$, so $\operatorname{Ker} \phi \subseteq J(R[[X]])$. Since $R \cong R[[X]] / \operatorname{Ker} \phi$, we obtain the result by Lemma 2.10 .

Given a ring $R$ and a bimodule ${ }_{R} M_{R}$, the trivial extension of $R$ by $M$ is the $\operatorname{ring} T(R, M)=R \oplus M$ with the usual addition and the following multiplication:

$$
\left(a_{1}, x_{1}\right)\left(a_{2}, x_{2}\right)=\left(a_{1} a_{2}, a_{1} x_{2}+x_{1} a_{2}\right) .
$$

This is isomorphic to the ring of all matrices $\left(\begin{array}{cc}r & m \\ 0 & r\end{array}\right)$, where $r \in R$ and $m \in M$ and the usual matrix operations are used.

Theorem 2.12. A ring $R$ is a generalized stable ring if and only if $T(R, M)$ is a generalized stable ring. 
Proof. From [13], we have $J(T(R, M))=T(J(R), M)$. We construct a map $\phi: R \longrightarrow T(R, M) / J(T(R, M))$ such that $\phi(x)=\overline{(x, 0)}$ for any $x \in R$. Suppose that $\overline{(x, m)} \in T(R, M) / J(T(R, M))$, then $\overline{(x, m)}=\overline{(x, 0)}+\overline{(0, m)}=\phi(x)$. Hence $\phi$ is a epimorphism and $\operatorname{Ker} \phi=\{x \in R \mid(x, m) \in J(T(R, M))\}=J(R)$. So $T(R, M) / J(T(R, M)) \cong R / J(R)$. So we complete the proof by Lemma 2.10 .

Now we consider the following subring of the triangular matrix ring. Let $R$ be a ring and let

$$
R_{n}=\left\{\left(\begin{array}{ccccc}
a & a_{12} & a_{13} & \cdots & a_{1 n} \\
0 & a & a_{23} & \cdots & a_{2 n} \\
0 & 0 & a & \cdots & a_{3 n} \\
\vdots & \vdots & \vdots & \ddots & \vdots \\
0 & 0 & 0 & \cdots & a
\end{array}\right) \mid a, a_{i j} \in R\right\}
$$

be a subset of the triangular matrix ring. Then $R_{n}$ is a ring with addition point-wise and usual matrix multiplication.

Proposition 2.13. A ring $R$ is a generalized stable ring if and only if $R_{n}$ is a generalized stable ring.

Proof. It is clear that

$$
J\left(R_{n}\right)=\left\{\left(\begin{array}{ccccc}
a & a_{12} & a_{13} & \cdots & a_{1 n} \\
0 & a & a_{23} & \cdots & a_{2 n} \\
0 & 0 & a & \cdots & a_{3 n} \\
\vdots & \vdots & \vdots & \ddots & \vdots \\
0 & 0 & 0 & \cdots & a
\end{array}\right) \mid a \in J(R), a_{i j} \in R\right\} .
$$

Since $R_{n} / J\left(R_{n}\right) \cong R / J(R)$, we obtain the result by Lemma 2.10 .

Let $R$ be a ring and let

$$
T_{n}(R)=\left\{\left(\begin{array}{ccccc}
a_{1} & a_{2} & a_{3} & \cdots & a_{n} \\
0 & a_{1} & a_{2} & \cdots & a_{n-1} \\
0 & 0 & a_{1} & \cdots & a_{n-2} \\
\vdots & \vdots & \vdots & \ddots & \vdots \\
0 & 0 & 0 & \cdots & a_{1}
\end{array}\right) \mid a_{i} \in R(1 \leq i \leq n)\right\}
$$

be a subset of the triangular matrix ring. Then $T_{n}(R)$ is a ring with addition point-wise and usual matrix multiplication.

Proposition 2.14. A ring $R$ is a generalized stable ring if and only if $T_{n}(R)$ is a generalized stable ring. 
Proof. It is clear that

$$
J\left(T_{n}(R)\right)=\left\{\left(\begin{array}{ccccc}
a & a_{2} & a_{3} & \cdots & a_{n} \\
0 & a & a_{2} & \cdots & a_{n-1} \\
0 & 0 & a & \cdots & a_{n-2} \\
\vdots & \vdots & \vdots & \ddots & \vdots \\
0 & 0 & 0 & \cdots & a
\end{array}\right) \mid a \in J(R), a_{i} \in R(2 \leq i \leq n)\right\} .
$$

Since $T_{n}(R) / J\left(T_{n}(R)\right) \cong R / J(R)$, we obtain the result by Lemma 2.10 .

Corollary 2.15. Let $R$ be a ring and $n$ any positive integer. Then $R$ is a generalized stable ring if and only if $R[x] /\left(x^{n}\right)$ is a generalized stable ring, where $\left(x^{n}\right)$ is the ideal generated by $x^{n}$.

Proof. Observe that $T_{n}(R) \cong R[x] /\left(x^{n}\right)$ for any positive integer $n$. Thus the result follows from Proposition 2.14.

Recall from [2], an idempotent $e \in R$ is left (resp. right) semicentral in $R$ if $e r e=r e$ (resp. ere $=e r$ ) for all $r \in R$. It is well known that if a ring $R$ has (weakly) stable range one, then $e R e$ has (weakly) stable range one for any $e^{2}=e \in R$. But we don't know the result whether or not true for generalized stable rings. For semicentral idempotents, we have the following result.

Theorem 2.16. Let $R$ be a generalized stable ring. Then eRe is also a generalized stable ring for any left (right) semicentral idempotent $e \in R$.

Proof. Given $a x+b=e$ with $a, x, b \in e R e$, then we have $(a+1-e)(x+$ $1-e)+b=1$. Since $R$ is a generalized stable ring, there exists $z \in R$ such that $a+1-e+b z=u \in K(R)$. Assume $s u t=1$ for some $s, t \in R$, that is, $s(a+1-e+b z) t=1$, thus $e s(a+1-e+b z) t e=e$. Since $e$ is a left semicentral idempotent, we have $(1-e) t e=0$, hence $e s(a+b z) t e=e$. In addition, we know that $a, b \in e R e$, thus ese $(a+b(e z e)) e t e=e$, that is, $a+b(e z e) \in K(e R e)$, as desired.

Similarly, we can prove $e R e$ is also a generalized stable ring for any right semicentral idempotent $e \in R$.

\section{References}

[1] P. Ara, G. K. Pedersen, and F. Perera, An infinite analogue of rings with stable rank one, J. Algebra 230 (2000), no. 2, 608-655.

[2] G. F. Birkenmeier, J. Y. Kim, and J. K. Park, Polynomial extensions of Baer and quasi-Baer rings, J. Pure Appl. Algebra 159 (2001), no. 1, 25-42.

[3] H. Y. Chen, Full elements in one-sided unit-regular rings, Comm. Algebra 33 (2005), no. $11,4115-4123$.

[4] _ Full elements in regular rings, Taiwanese J. Math. 8 (2004), no. 2, 203-209.

[5] __ Generalized stable exchange rings, Algebra Colloq. 15 (2008), no. 2, 193-198.

[6] ——, Generalized stable regular rings, Comm. Algebra 31 (2003), no. 10, 4899-4910.

[7] - On exchange $Q B_{\infty}$-rings, Algebra Colloq. 14 (2007), no. 4, 613-623.

$[8] \_$, On exchange QB-rings, Comm. Algebra 31 (2003), no. 2, 831-841. 
[9] _ On generalized stable rings, Comm. Algebra 28 (2000), no. 4, 1907-1917.

[10] _ On QB endomorphism rings, Comm. Algebra 35 (2007), no. 12, 4005-4017.

$[11] \_$, On $Q B_{\infty}$-rings, Comm. Algebra 34 (2006), no. 6, 2057-2068.

[12] W. K. Nicholson, Lifting idempotents and exchange rings, Trans. Amer. Math. Soc. 229 (1977), 269-278.

[13] W. K. Nicholosn, J. K. Park, and M. F. Yousif, Extensions of simple-injective rings, Comm. Algebra 28 (2000), no. 10, 4665-4675.

[14] J. Q. Wei, Unit-regularity and stable range conditions, Comm. Algebra 33 (2005), no. 6, 1937-1946.

Department of Mathematics

HeXi UNIVERSITY

Zhangye, 734000, P. R. China

E-mail address: zhangwru@163.com 